\title{
MADERA DE COMPRESIÓN EN PINUS RADIATA D. DON: I, CARACTERÍSTICAS ANATÓMICAS
}

\section{COMPRESSION WOOD IN RADIATA PINE: I, ANATOMICOL FEATURES}

\author{
J.E.Diaz-vaz ${ }^{l}$; A.Fernandez ${ }^{1}$; L.Valenzuela ${ }^{2}$; M.Torres ${ }^{l}$
}

\section{RESUMEN}

Se caracterizó la madera de compresión en 10 árboles de Pinus radiata D.Don con inclinaciones menores a $10^{\circ}$ en dos sitios.

Los diámetros de traqueidas de los árboles del sitio Arena húmeda resultaron estadísticamente mayores que los del sitio Arcilla; pero no resultaron diferentes al comparar madera juvenil y madura o al comparar madera de compresión con madera opuesta.

Diferencias significativas se determinaron en el largo de traqueida entre sitios (Arena húmeda-Arcilla), tipo de madera (madera de compresión-opuesta) y edad (madera juvenil-madura), resultados coincidentes con otros autores.

Para el coarseness diferencias no significativas entre sitios, así como entre madera juvenil y madura y entre madera de compresión y opuesta coinciden y difieren de los de otros autores que comparan árboles de distintos sitios.

Las discrepancias y coincidencias de estos resultados con los de otros autores se explican considerando el comportamiento diferente de las dimensiones celulares de la madera temprana y tardía y las diferencias que hay entre madera de compresión leve y la madera de compresión severa.

Palabras claves: Fustes inclinados, madera de compresión leve, largo de traqueidas, pino radiata, coarseness, diámetros de traqueidas, madera opuesta, madera juvenil, madera madura.

\section{ABSTRACT}

Compression wood in 10 Pinus radiata D. Don trees with lean smaller to $10^{\circ}$ from two sites were characterized. The tracheid diameters of the trees of the humid sand site were statistically greater than those from the clay site; but they were not different when comparing juvenile wood with mature wood or to it compares compression wood with opposite wood.

Significant differences were determined in the tracheid length between sites (humid sand-clay), type of wood (compression-opposite wood) and age (juvenile-mature wood), were similar results with other authors.

For coarseness non significant differences between sites, as well as between juvenile and mature wood and compression and opposite and agree and different from those of other authors who compare trees of different sites.

The discrepancies and coincidences of these results with those of other authors are explained considering the behavior from the cellular dimensions of the earlywood and latewood wood and the differences between severe compression and mild compression wood.

Keywords.Lend stems, mild compression wood, tracheid length, pino radiata, coarseness, tracheid diameter, opposite wood, earlywood, laterwood.

\footnotetext{
${ }^{1}$ Instituto de Tecnología de Productos Forestales, Universidad Austral de Chile. Valdivia, Chile. jdiazvaz@uach.cl

${ }^{2}$ Facultad de Ciencias Forestales, Universidad de Concepción. Concepción, Chile. 1valenzu@udec.cl 


\section{INTRODUCCION}

Las singularidades y propiedades de la madera de compresión (MC) son diferentes a las que se encuentran en la madera opuesta e igualmente distintas a las de los árboles que no tienen madera de compresión. Generalmente este tipo de madera se relaciona con árboles inclinados y curvados. Sin embargo, en rodales con árboles que crecen sin inclinación o con inclinaciones menores, también se puede encontrar madera de compresión (MC).

Distintos estudios demuestran las diferencias entre la madera de compresión (MC), la madera opuesta y la madera que no es de compresión en Pinus radiata D. Don y otras especies de coníferas. Entre las diferencias más notables se tiene: mayor proporción de lignina, falta de la pared $\mathrm{S}_{3}$, presencia de fracturas helicoidales, mayor ángulo fibrilar en la pared $\mathrm{S}_{2}$, secciones de las traqueidas redondeadas, presencia de espacios intercelulares, reducción de la proporción de lignina en la lámina media y concentración de este compuesto entre la $\mathrm{S}_{1}$ y $\mathrm{S}_{2}$, menor largo de traqueidas, mayor ancho de la pared celular en la madera intermedia y tardía, anillos más anchos, coloración más oscura y cambios dimensionales longitudinales mayores a los de la madera opuesta o a la que no es de compresión. La excentricidad del crecimiento es también una singularidad que presentan muchos árboles con MC. (Burdon 1975; Harris 1977; Nicholls 1982; Timell 1986; Vargas y Diaz-vaz 1992; Singh y Donaldson 1999; Lomagno y Rozas 2001; Diaz-vaz 2003; Donaldson et al. 2004; Schmitt et al. 2005).

En general, las singularidades de la MC implican efectos no siempre aceptables sobre los productos que se fabrican con esta madera ya que afecta a: procesos de pulpaje, comportamiento físico y mecánicos de tableros de fibras, de tableros de partículas, incide sobre la permeabilidad de la madera y en las curvas de secado, afecta la calidad de la madera aserrada y sus productos (du Torr 1963; Nicholls 1982; Öhman 1999; Davis et al. 2002; Akbulut et al. 2004; Ban et al. 2004; Lindstom et al. 2004; Roffael et al. 2005)

La MC se presenta con distintos grado de severidad, esto es, MC de grado severo, MC de grado leve y la zona que se conoce como madera opuesta y que es la se encuentra en esos árboles pero no posee características de MC. En el caso de esta última, madera opuesta, sólo se encuentran diferencias menores en comparación con la madera de árboles sin MC.

Las características de la MC varían significativamente dependiendo del grado que ella presenta. $\mathrm{La}$ MC leve presenta diferencias menores con la madera opuesta respecto a: presencia de espacios intercelulares, dimensiones celulares, densidad de la madera y ángulo fibrilar. Se caracteriza por una reducida lignificación de la lámina media, primer indicio de la formación de este tipo de MC.

Investigaciones indican una deslignificación de la lámina media en los vértices de las células, lo que correspondería a una característica típica de la MC leve en madera juvenil de

Pinus radiata. También la reducida concentración de lignina, de la parte externa de la pared secundaria $\left(\mathrm{S}_{2 \mathrm{~L}}\right)$, presente en la zona de compresión leve, se va incrementando hasta que se llega a la concentración máxima en la zona con compresión más severa.

La información que se maneja indica que árboles con inclinaciones menores pueden tener mayor proporción de MC leve. En estas maderas las diferencias en propiedades y singularidades respecto a la madera opuesta y a la madera que no es de compresión son reducidas. Lo mismo sucede con los productos que con ella se fabrican.

Las diferencias de las singularidades de la MC se asocian no solo al grado de leve a severo sino que también hay diferencias entre la madera de compresión de la madera juvenil y la madera madura. Incluso hay diferencias entre la madera de compresión de la madera temprana y las de la madera tardía. 
Considerando que las propiedades y singularidades de la madera de árboles poco inclinados corresponden frecuentemente a MC leve, con modificaciones menores respecto a la madera opuesta, es que el presente estudio analiza comparativamente las características anatómicas de la madera de compresión y la respectiva madera opuesta, en árboles sin inclinaciones mayores, pertenecientes a dos rodales de Pinus radiata, creciendo en diferentes sitios.

\section{MATERIAL Y MÉTODO}

Se tomaron muestras de 10 árboles $P$. radiata de 22 años de edad de dos rodales, uno establecido en suelo de arena húmeda en la provincia de Bío Bío y el otro en suelo arcilloso de la provincia de Malleco. Los árboles elegidos eran de fustes rectos, exentos de defectos y con inclinaciones no mayores a $10^{\circ}$ respecto a la vertical, inclinación normal presente en los árboles de los dos rodales estudiados.

De cada árbol se extrajo rodelas de tres niveles de altura en el fuste. Las rodelas del tercio basal se obtuvieron a la altura del pecho (DAP) y la del segundo y tercio superior se tomaron centradas en el tercio respectivo, esto es, entre la altura de pecho y una altura aprovechable de $10 \mathrm{~cm}$ de diámetro mínimo.

En las rodelas se identificó, de manera visual y sobre las superficies pulimentadas, las zonas de madera de compresión y de madera opuesta. La madera de compresión se reconoció mediante: por excentricidad de la médula, diferencias del ancho de anillo, por la coloración que presentaba la madera y también por la apariencia de madera tardía que presenta la madera intermedia.

En cada rodela, se seleccionó una cantidad de madera opuesta, igual y de los mismos anillos de la considerada como MC. De este modo, ellas participaron en proporción similar y con edades fisiológicas idénticas en todos los ensayos realizados en cada rodela.

Los dos tipos de maderas discriminadas, esto es de compresión y opuesta, se identificaron tanto para madera juvenil como para madera madura. Se consideró como madera juvenil aquella comprendida desde la médula hasta el décimo anillo.

En la MC y en la madera opuesta se realizaron determinaciones de las dimensiones de las traqueidas. Las mediciones se efectuaron en tres alturas cuando fue posible. Se diferenció cada vez la madera juvenil de la madera madura.

Las determinaciones de largo de traqueidas y diámetro de traqueidas se realizaron mediante digitalización de imágenes de traqueidas maceradas, en una tableta digitalizadora, provista de un software según Diaz-vaz y Morales. Por su parte, el coarseness se midió siguiendo el procedimiento descrito en la norma TAPPI t $234 \mathrm{~cm}-84$.

Las mediciones se realizaron sobre traqueidas maceradas predominantemente de madera tardía. En ella las células cortas y las largas de esta madera tardía tienen igual probabilidad de resistir la acción maceradora sin romperse. Así las dimensiones promedios que se obtienen representan mejor tanto a traqueidas largas como a traqueidas cortas (Diaz-vaz 2003). 


\section{RESULTADOS}

Previo a realizar los análisis estadísticos se verificó la normalidad de los datos según ShapiroWilks y la homogeneidad de varianza según Bartlett (Morales 2005). Aquellos datos que no se mostraron normales o con varianzas no homogéneas debieron transformarse. Este fue el caso del diámetro celular

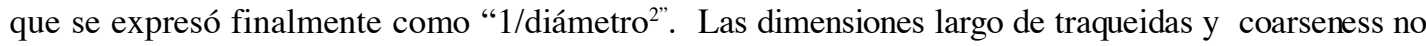
requirieron transformación.

Se compararon los resultados obtenidos de las maderas de los dos sitios, de la madera de compresión y opuesta y de la madera juvenil y madura para los casos del diámetro de traqueidas, el largo de traqueidas y el coarseness.

\section{Diámetro de traqueidas (1/diámetro $\left.{ }^{2}\right)$.}

En la figura 1 y tabla 1 se resume los resultados calculados para el diámetro de traqueidass en ambos sitios. Ellos resumen las diferencias entre madera juvenil y madura tanto en madera de compresión (MC) como en madera opuesta, en las diferentes alturas en el fuste.

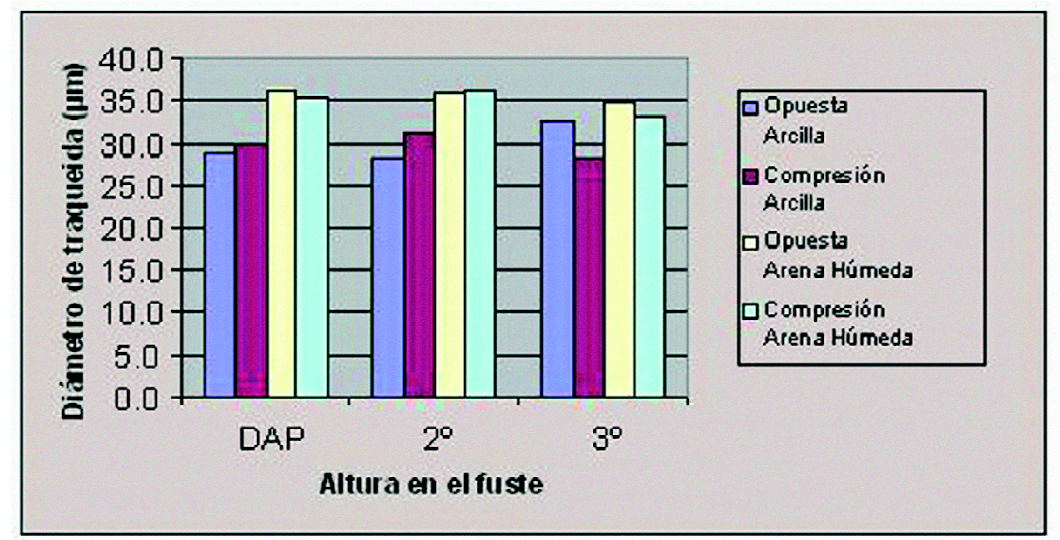

Figura 1 : Diámetro de traqueidas de madera de compresión y opuesta ( $\mu \mathrm{m})$.

Madera juvenil de los dos sitios.

Los promedios del diámetro de traqueidas, en algunos de los casos parecen indicar valores diferentes entre la MC y la madera opuesta. Sin embargo el análisis por separado para cada altura, mostró sólo un caso en la que la MC tuvo un diámetro de traqueidas menor que la madera opuesta. Este correspondió al sitio Arcilla, a la altura del tercer tercio, de la madera juvenil, en donde los diámetros de las traqueidas en la MC resultaron menores y estadísticamente significativos, a los de la madera opuesta, según Duncan a un nivel de confianza del 95,0 \% . 
Tabla 1 : Diámetro de traqueidas de madera de compresión y opuesta $(\mu \mathrm{m})$.

Madera madura de los dos sitios.

\begin{tabular}{|c|c|c|c|c|}
\hline \multirow{2}{*}{$\begin{array}{l}\text { AT TURA } \\
\text { EN EL } \\
\text { FUSIE }\end{array}$} & \multicolumn{2}{|c|}{$\triangle R C L L \Lambda$} & \multicolumn{2}{|c|}{ ARENA HÚMEDA } \\
\hline & Opuesta & Compresión & Opuesta & Compresión \\
\hline DAP & 29,4 & 28,0 & 34,8 & 33,5 \\
\hline $2^{\circ}$ & 33,8 & 31,5 & & \\
\hline
\end{tabular}

El efecto sobre el diámetro de traqueidas de los factores: sitio (Arcilla y Arena húmeda), edad (madera juvenil y madura), y tipo de madera (compresión y opuesta), se probaron en un ANOVA para un nivel de confianza del 95,0\% (ver tabla 2).

Los resultados indican que las traqueidas de las maderas del sitio Arena húmeda tuvieron diámetros significativamente mayores que las del sitio Arcilla (Ver tabla 2).

El efecto de la edad según el análisis de varianza, indicó que en ambos sitios las diferencias, entre los valores de diámetros de las traqueidas de la madera juvenil y los de madera madura, no fueron estadísticamente significativas.

Tabla 2 : Análisis de varianza para diámetro de traqueidas $\left(1 /\right.$ diámetro $\left.^{2}\right)$

\begin{tabular}{|l|l|l|l|l|}
\hline Fuente & $\begin{array}{l}\text { Suma de } \\
\text { cuadrados }\end{array}$ & GL & F & P-Valor \\
\hline Efectos principales & & & & \\
\hline A: Sitio & 0,00000179419 & 1 & 52,16 & 0,0000 \\
\hline B: Madera & $8,44318 \mathrm{E}-8$ & 1 & 2,45 & 1,1209 \\
\hline C: Edad & $1,29451 \mathrm{E}-8$ & 1 & 0,38 & 0,5412 \\
\hline & & & & \\
\hline RESDUOS & 0,00000288945 & & & \\
\hline TOTAL(corregido) & 0,00000478709 & & & \\
\hline
\end{tabular}

Un resultado similar al anterior se tuvo para los diámetros de traqueidas maceradas, preferentemente de madera tardía (Diaz-vaz 2003), las que no mostraron diferencias estadísticamente significativas al comparar los diámetros en la madera de compresión con los de la madera opuesta.

\section{Largo de traqueidas.}

Los resultados del largo de traqueidas se resumen en la figura 2 y en la tabla 3. Los largos de traqueidas en la madera de compresión fueron mayores que los registrados en la madera opuesta. Las diferencias fueron notorias especialmente en el sitio Arcilla. Los resultados obtenidos en los árboles del sitio Arena húmeda no mostraron diferencias tan notorias como los registrados en el sitio Arcilla. 


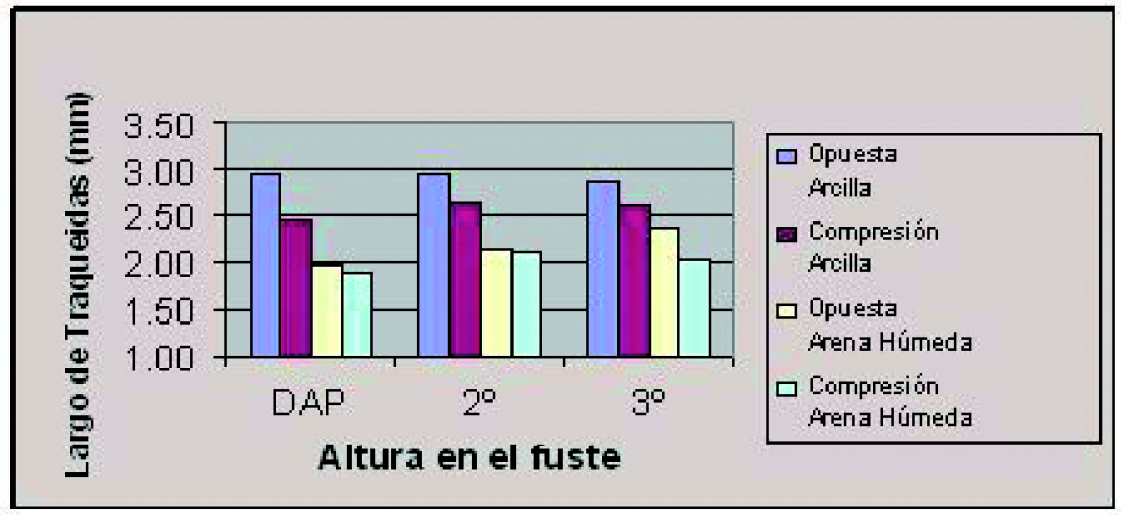

Figura 2: Largo de traqueidas de madera de compresión y opuesta (mm). Madera juvenil de los dos sitios.

Tabla 3 : Largo traqueidas de madera de compresión y opuesta $(\mathrm{mm})$. Madera madura de los dos sitios.

\begin{tabular}{|l|c|c|c|c|}
\hline \multirow{2}{*}{ ALTURA } & \multicolumn{2}{|c|}{ ARCILLA } & \multicolumn{2}{c|}{ ARENA HÚMEDA } \\
\cline { 2 - 5 } EN EL & Opuesta & Compresión & Opuesta & Compresión \\
\hline DAP & 3,28 & 2,92 & 2,50 & 2,30 \\
\hline $2^{\text {o }}$ & 3,62 & 3,04 & & \\
\hline
\end{tabular}

El análisis de varianza del tabla 4 muestran el efecto de los factores: sitio (Arcilla y Arena húmeda), edad (madera juvenil y madura), y tipo de madera (compresión y opuesta), sobre el largo de las traqueidas, para un nivel de confianza del $95.0 \%$. Todos los factores tuvieron un efecto estadísticamente significativo en el largo de traqueidas para ese nivel de confianza.

Tabla 4 : Análisis de varianza para el largo de traqueidas.

\begin{tabular}{|l|l|l|l|l|}
\hline Fuente & $\begin{array}{l}\text { Suma de } \\
\text { cuadrados }\end{array}$ & GL & F & P-Valor \\
\hline Efectos principales & & & & \\
\hline A: Sitio & 10,433 & 1 & 152,82 & 0,0000 \\
\hline B: Madera & 1,9537 & 1 & 28,62 & 0,0000 \\
\hline C: Edad & 3,26991 & 1 & 3,26991 & 0,0000 \\
\hline & & & & \\
\hline RESDUOS & 5,66642 & & & \\
\hline TOTAL(conegido) & 23,0207 & & & \\
\hline
\end{tabular}


El análisis indica que el largo de las traqueidas, en general, resultó mayor en las maderas del sitio Arcilla que en las maderas del sitio Arena húmeda. En la madera opuesta, las traqueidas también fueron mayores que las de MC. Igualmente, como era de esperar, las traqueidas de la madera madura fueron más largas que las traqueidas de madera juvenil.

Considerando separadamente los dos sitios y las tres alturas, los largos de traqueidas, resultan significativamente diferentes a la altura del DAP y en el segundo tercio del sitio Arcilla, según contraste múltiple de rangos de Duncan. Por el contrario, en el sitio Arena húmeda y en el tercer tercio del sitio Arcilla de la madera juvenil, las diferencias no resultaron significativas.

El efecto de la edad sobre el largo de traqueidas y el tipo de madera (MC y madera opuesta) resultaron significativos según el análisis de varianza aplicado (ver tabla 4).

\section{Coarseness}

El coarseness definido como el peso por unidad de largo de las traqueidas, tiene una estrecha relación con la densidad de la madera y es, de entre las dimensiones de las traqueidas, un muy buen índice para predecir las propiedades de pulpas. Sin embargo, son pocos los trabajos que incluyen este tipo de determinación.

Los valores promedios calculados para coarseness de los árboles de los dos sitios se muestran en la figura 3 y en el tabla 5. Las diferencias entre la MC y la madera opuesta no muestran tendencias consistentes en ambos sitios. Igual resultado se tiene al comparar las distintas alturas en el fuste (ver figura 3 y tabla 5). Tampoco se obtuvieron diferencias del coarseness entre la madera juvenil y la madura.

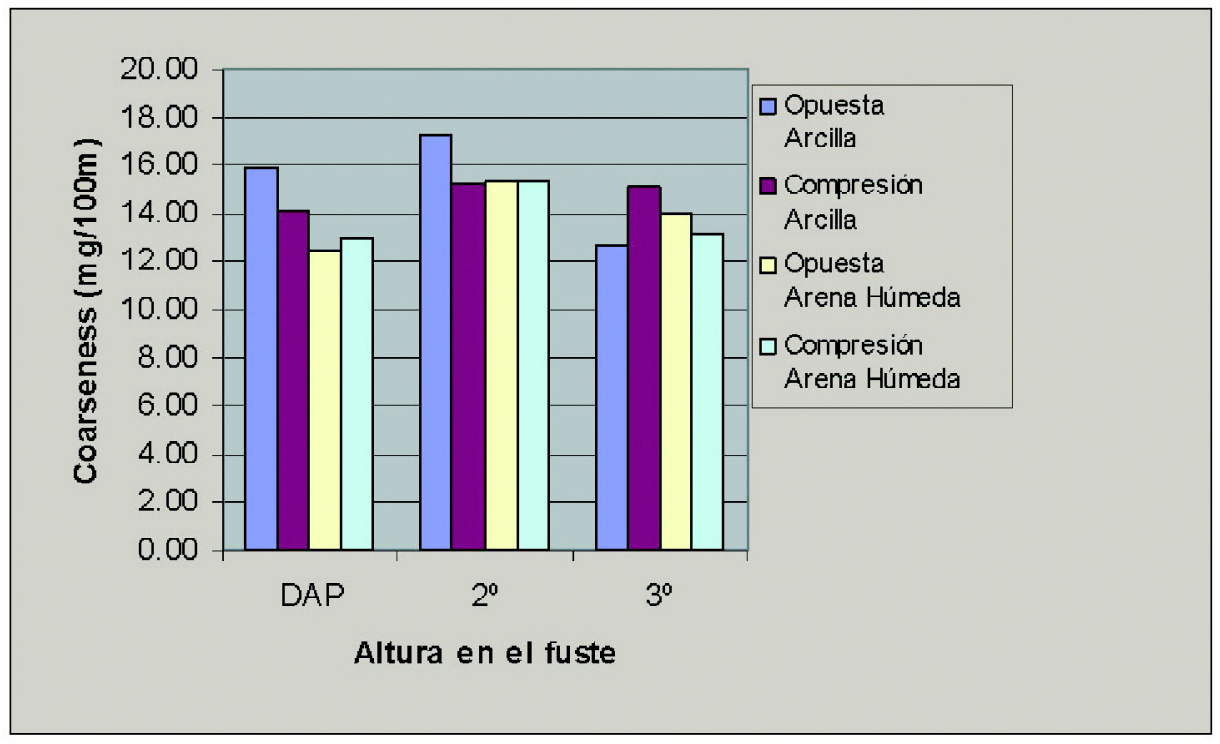

Figura 3 : Coarseness en madera de compresión y opuesta (mg/100m). Madera juvenil de los dos sitios 
Tabla 5 : Coarseness en madera de compresión y opuesta (mg/100m).

Madera juvenil de los dos sitios

\begin{tabular}{|l|r|r|r|r|}
\hline ALTURA & \multicolumn{2}{|c|}{ ARCILLA } & \multicolumn{2}{|c|}{ ARENA HÚMEDA } \\
EN FI. & \multicolumn{1}{|c|}{ Opuesta } & Compre & Opuesta & Compre \\
\hline FUSTE & 13,72 & 15,82 & 15,45 & 15,88 \\
\hline DAP & 15,91 & 15,65 & & \\
\hline $2^{\circ}$ & & & \\
\hline
\end{tabular}

El análisis de varianza con un nivel de confianza del $95.0 \%$, confirmó que ninguno de los factores estudiados: sitio (Arcilla y Arena húmeda), edad (madera juvenil y madura), y tipo de madera (compresión y opuesta), tuvo efecto sobre los valores del coarseness (ver tabla 6).

Tabla 6: Análisis de varianza para el coarseness.

\begin{tabular}{|l|l|l|l|l|}
\hline Fuente & $\begin{array}{l}\text { Suma de } \\
\text { cuadrados }\end{array}$ & GL & F & P-Valor \\
\hline Efectos principales & & & & \\
\hline A: Sitio & 13,8121 & 1 & 1,29 & 0,2599 \\
\hline B:Madera & 6,66367 & 1 & 0,62 & 0,4330 \\
\hline C:Edad & 17,9376 & 1 & 1,67 & 0,1997 \\
\hline & & & & \\
\hline RESDUOS & 945,312 & & & \\
\hline TOTAL(corregido) & 980,369 & & & \\
\hline
\end{tabular}

\section{DISCUSION}

La importancia de estudiar la madera de compresión (MC) se fundamenta en las singularidades diferentes que esta madera presenta en comparación con la madera opuesta y la madera que no es de compresión y el efecto que tiene sobre los productos fabricados con esta madera.

Sin embargo, no siempre es fácil detectar su presencia y determinar su incidencia asociándola a la inclinación de árboles. Esta dificultad se podría deber entre otras razones, a que no se distinga entre grados de MC leve y MC severa (Low 1964; Nicholls 1982).

En Pinus radiata árboles rectos e inclinados tienen la tendencia a producir crecimiento excéntrico suave, con ello médula excéntrica y formación de MC, la cual se encuentra mayoritariamente en la madera tardía siendo del tipo de MC leve (Nicholls 1982). También en esta especie se puede presentar MC leve sin que ella esté limitada a un solo lado de la sección, como es en la mayoría de casos de la MC severa (Harris 1977).

\section{Diámetro de traqueidas (1/diámetro $\left.{ }^{2}\right)$.}

Algunos autores indican que la MC leve no muestra diferencias significativas de diámetros celulares, ancho de pared celular ni densidad, cuando se compara con la madera opuesta (Donaldson et al. 2004) y se indica también que la MC leve es más difícil de detectar a simple vista (Andersson y Walter 1995). Por el 
contrario, algunos estudios realizados con microscopía electrónica en MC leve de Pinus radiata, sugieren que algunas singularidades de la MC leve son diferentes a las que no son de compresión y que por ello esta madera afectan a los productos que la utilizan (Singh 1997; Singh y Donaldson 1999).

Todo lo anterior deja a la MC y por ello a la del tipo leve como un tema relevante en la investigación de las singularidades anatómicas que ella presenta.

Los resultados obtenidos en el presente estudio de $\mathrm{MC}$ en árboles de inclinación menor, indicaron diferencias en los diámetros de traqueidas entre los sitios. En Arena hœmeda resultaron mayores que los registrados en el sitio Arcilla. Estas diferencias difierencon lo determinado en Pinus radiata en Chile (Melo et al. (1988) pero son coincidentes con otros estudios realizados en Pinus radiata en diferentes sitios (Burdon 1975; Nyakuengama et al. 2003).

Al comparar los diámetros de las traqueidas de la madera juvenil con la madera madura, no existieron diferencias estadísticamente significativas. Este resultado es coincidente con lo informado por Melo et al. (1988) para Pinus radiata de Chile y contrasta con lo informado también para madera de Pinus radiata en la que se reconoce un incremento de los diámetros de las traqueidas desde la médula a la corteza (Cown 1975). También en Pinus taeda, el diámetro tangencial aumentó en los primeros $100 \mathrm{~mm}$ desde la médula (Jones et al. 2005).

Estas discrepancias se podrían explicar considerando que las determinaciones que se informan se realizaron principalmente sobre madera tardía. Al respecto algunos estudios indican incrementos relevantes del diámetro de traqueidas con aumentos de la edad del cambium, en el caso de la madera temprana. Pero esa tendencia no se registra en el caso de la madera tardía, segœn lo mencionado por Schwarz ya en el año 1899 para Pinus sylvestris (Trendelenburg y Mayer-Wegelin 1955). Por lo anterior el efecto de la edad en determinaciones realizadas con madera temprana pueden dar resultados diferentes a los que se obtienen con mediciones en la madera tardía.

Lo anterior puede concluirse también considerando el caso de madera de Picea abies en la cual los diámetros radiales de traqueidas de la madera temprana fueron dependientes de la edad del cambium, del ancho de anillo y del sitio $\left(\mathrm{r}^{2}=0,67\right)$. Por el contrario, el efecto de la edad del cambium sobre el diámetro radial de traqueidas de la madera tardía, incluido el sitio, resultó muy reducido $\left(\mathrm{r}^{2}=0,19\right)$ (Lindström 1997).

Las diferencias de los diámetros celulares, entre la MC y la madera opuesta, no resultaron significativas segœn comparaciones múltiples de Duncan. Para analizar este resultado debe tenerse presente que las mediciones corresponden a traqueidas maceradas de madera tardía, según lo propuesto por Diaz-vaz (2003).

Las discrepancias y coincidencias de estos resultados con los de otros autores no solo requiere considerar, si se trata de madera temprana o tardía sino que también si se trata de MC leve o MC severa. En ambos casos el comportamiento de los diámetros celulares es diferente.

En el caso de madera temprana de Picea abies el diámetro de las traqueidas de madera opuesta resulta mayor que las de madera de compresión (Mayr y Cochard 2003). Igual resultado determinaron Spicer y Gartner (1998) en traqueidas de madera temprana con madera opuesta, en ramas de Pseudotsuga menziesii. Por el contrario, en este mismo estudio, las traqueidas de la madera tardía de la MC no diferían del diámetro de las traqueidas de la madera opuesta.

Por otra parte las diferencias que existen entre MC leve y MC severa pueden explicar las diferencias del diámetro de traqueidas. En Pinus radiata las dimensiones transversales de traqueidas de la MC leve, no resultaron diferentes a la madera opuesta. Pero que en el caso de la MC severa, las diferencias fueron significativas, especialmente en el caso de la madera tardía (Donaldson et al. 2004). 
También comparaciones de las características morfológicas transversales de traqueidas de $\mathrm{MC}$, no mostraron diferencias cuando se compararon traqueidas de madera normal con las de $\mathrm{MC}$, en madera de Pinus radiata. Probablemente la MC en ese estudio puede haber correspondido a MC del tipo leve. En ese mismo estudio si se encontró diferencias entre MC y madera opuesta en el caso de la madera de Picea sitchensis (Moëll y Fujita 2004).

\section{Largo de traqueidas.}

Los valores promedios determinados en este estudio para el largo de traqueidas, correspondieron al rango conocido para la especie creciendo en Chile (Delmastro et al., 1982; Melo et al., 1988; Martínez et al. 1989; Diaz-vaz et al., 1989).

Las diferencias significativas que se registraron entre los dos sitios concuerdan también con lo informado por diversos autores (Melo et al. 1988; Nyakuengama et al. 2003; Dúchense y Zhang 2004; Moltenberg, M $\varnothing \mathrm{ib} \varnothing 2006)$

El largo de traqueidas resultó afectado, además del sitio, por la edad. Este efecto está asociado a la actividad periódica del cambium y es especialmente importante en los primeros años. Luego de ciertos años de actividad del cambium, las traqueidas se mantienen con un largo promedio. En algunas especies de coníferas como Fitzroya cupressoides el aumento de las traqueidas puede durar hasta cerca de los 100 años (Diaz-vaz 2003). En cambio en coníferas de rápido crecimiento como en árboles de Pinus radiata, el aumento se produce en los primeros 7 a 11 años aproximadamente (Rivas 1982). Un valor similar se determinó en Pseudotsuga menziesii, mediante análisis de cluster, con un incremento hasta los 8 a 11 años (Diaz-vaz 1981)

Las diferencias del largo de traqueidas entre la madera juvenil y la madura resultantes en este estudio fueron coincidentes con la mayoría de las investigaciones realizadas al respecto (Cown y Mc Conchie 1980; Uprichard 1980; Melo et al. 1988; Martínez et al., 1989; Lindstrom 1997).

En la MC el mayor crecimiento secundario en diámetro se asocia al aumento de las divisiones anticlinales pseudotransversales de las iniciales fusiformes del cambium. Es así como el mayor nœmero de divisiones anticlinales en la MC implica una reducción en las dimensiones celulares de las traqueidas que se forman en la MC (Yoshizawa et al., 1987). De allí que diferencias del largo de traqueidas entre MC y madera opuesta deberían resultar similares al considerar madera temprana o madera tardía.

El largo de traqueidas de la madera temprana y también el de la madera tardía resulta menor en la $\mathrm{MC}$ respecto a la madera opuesta en todos los grados de MC. Al comparar MC leve y MC de grado medio a severo se ha informado de algunas diferencias entre madera temprana y tardía. Las mayores diferencias entre madera opuesta y de compresión se presentaron en la madera tardía de MC severa (Shelbourne y Ritchie 1968). Esto œltimo, puede hacer necesario considera que los resultados aquí informados pudieran ser algo menores si se hubiera incluido en las maceraciones madera temprana.

Teniendo presente lo anterior, los resultados registrados coinciden con lo determinado en diferentes especies en los cuales la MC leve estudiada tiene traqueidas más cortas que la madera opuesta. Es importante considerar que traqueidas más cortas, como las de MC, que además son frágiles y quebradizas pueden producir papeles de resistencias mecánicas reducidas (Zobel, 1981). 


\section{COARSENESS}

Los valores determinados para coarseness coinciden con los determinados por Melo et al. (1987) para la misma especie, quienes no encontraron ordenación o tendencia alguna en relación con la procedencia del material creciendo en diferentes sitios en Chile. En otras especies tales como Picea glauca (Dúchesne y Zhang 2004) y en Tsuga heterophylla (Watson et al. 2003) tampoco se han encontrado diferencias de coarseness entre sitios.

Contrario a los resultados obtenidos y a los otros estudios mencionados en el párrafo anterior, se informa de diferencias del coarseness entre sitios similares a los del presente estudio, en Pinus radiata, que pudieran ser significativas (Martínez et al. 1989). También en Pinus radiata se indican diferencias entre sitios Nyakuengam et al. (2003). En Eucalyptus globulus y en E. nitens también se han informado diferencias de coarseness entre sitios (Muneri y Raymond, 2001). Mediciones del coarseness que consideren sólo madera tardía, caso del presente estudio, pudieran entregar resultados distintos a los de otros autores.

Los valores de coarseness registrados no resultaron diferentes cuando se comparó la madera juvenil con los de la madura, segœn el análisis de varianza. Este resultado no coincide con determinaciones efectuadas en Pinus radiata que muestran diferencias con la edad (Melo et al 1988; Martínez et al. 1989; Schimleck y Evans 2004). Un efecto pequeño pero consistente, se determinó para el coarseness y la densidad (que están correlacionados) con la edad en Pinus radiata (Uprichard 2002). Por el contrario un resultado similar al determinado en el presente estudio se informó para Tsuga heterophylla especie en la que no se detectó un efecto significativo de la edad en los valores de coarseness (Watson et al. 2003).

Los valores de coarseness no diferentes entre madera juvenil y madura, podrían interpretarse como un comportamiento diferente de las traqueidas de madera tardía respecto a lo que acontece en madera temprana. De acuerdo a los resultados de Evans (1994) en Pinus radiata, la relación entre densidad de la madera y coarseness, existe en madera temprana, pero no se presenta en el caso de la madera tardía. De allí que un incremento de densidad de la madera con la edad no necesariamente significaría incrementos del coarseness con la edad.

Los resultados obtenidos indican también, según el análisis de varianza aplicado, que las diferencias entre la MC y la madera opuesta tampoco resultaron significativas para el coarseness.

\section{CONCLUSIONES}

En los dos sitios estudiados los árboles de Pinus radiata, con inclinaciones menores de no más de $10^{\circ}$, presentaron características en sus traqueidas de madera tardía comparables a las de madera de compresión del tipo leve.

Los diámetros de traqueidas del sitio Arena húmeda resultaron mayores que los del sitio Arcilla. Diferencias coincidentes con otros estudios realizados en Pinus radiata y contrarios a otros para la especie.

Al comparar la madera juvenil con la madera madura, los diámetros de las traqueidas no presentaron diferencias estadísticamente significativas. Resultado que coincide con lo informado para Pinus radiata de Chile pero que contrastan con lo informado en otros estudios también en madera de Pinus radiata. Estas discrepancias se han explicado considerando que las determinaciones se refieren a madera tardía y que para esta madera otros autores informan que no se han encontrado diferencias. 
Las diferencias de diámetros celulares entre MC y madera opuesta no resultaron significativas para este caso de traqueidas de madera tardía y MC leve. Las discrepancias y coincidencias de estos resultados con los de otros estudios, resalta el comportamiento diferente de los diámetros celulares dependiendo del tipo de madera estudiado, esto es, madera temprana o tardía e igualmente MC leve o MC severa.

Los valores promedios del largo de traqueidas y las diferencias entre sitios se inscriben dentro de lo esperado para la especie creciendo en Chile. La variabilidad del largo de traqueidas resultó afectada como era de esperar por el sitio, el tipo de madera y la edad.

Las diferencias del largo de traqueidas entre la madera juvenil y la madura coincidió con la mayoría de las investigaciones realizadas al respecto. Las traqueidas de la madera temprana y tardía resultaron menores en esta MC respecto a la madera opuesta.

Para el coarseness diferencias no significativas entre sitios coinciden y difieren de los de otros autores que comparan árboles de distintos sitios. Las diferencias no significativas encontradas al comparar el coarseness de la madera juvenil y el de la madera madura se pueden deber a que las determinaciones correspondieron a traqueidas de madera tardía en la que incrementos con la edad no necesariamente significan incrementos del coarseness.

La MC estudiada registró valores de coarseness similares a los determinados en la madera opuesta.

\section{NOTA}

* Proyecto financiado con apoyo del Comité de Investigaciones Básicas, CMPC Fábrica Laja, Chile.

\section{BIBLIOGRAFIA}

AKBULUT, T.; AYRILMIS, N. ; KOC, E. 2004. Influence of compression wood on physical and mechanical properties of medium density fiberboard. Wood Research 49(3):17-24.

ANDERSSON, C.; WALTER, F.; 1995. Classification of compression wood using digital image analysis. For. Prod. J. 45: 87-92

BAN, W.; MANCOSKY, D.; LUCIA, L. 2004.

Evaluation of the pulping response of juvenil and mature black spruce compression wood. Cellulose Chemistry and Technology, 38(1-2): 79-85.

BURDON, R. 1975. Compression wood in Pinus radiata on four different sites. N. Z.J.For. Sci. 5(2):152-164

COWN, D. 1975. Variation in tracheid dimensions in the stem of 26 year-old radiata pine Tree. Appita 28(4):237-245

COWN, D.; McCONCHIE, D. 1980. Wood property variations in an old-crop stand of radiata pine. New Zealand Journal of Forestry Science 10(3):508-520.

DAVIS, C.; CARRINGTON, C.; SUN, Z. 2002. The influence of compression wood on the drying curves of Pinus radiata dried in dehumidifier conditions. Drying Technology 20 (10): 2005-2026. 
DELMASTRO, R.; DIAZ-VAZ, J.E.; SCHLATTER, J. 1982. Variabilidad de las características hereditarias del Pinus radiata D. Don. Documento de Trabajo NY 43 CONAF/PNUD/FAO. Santiago de Chile 89 pp.

DIAZ-VAZ, J.E. 1981. Delimitación de madera temprana-tardía y madera juvenil madura en pino oregón. Bosque 4(1):55-58.

DIAZ-VAZ, J.E.; POBLETE, H.; JUACIDA, R.; DEVLIEGER, F. 1989. Maderas comerciales de Chile. Chilean comercial woods. Marisa Cœneo Ediciones, Chile 91 p.

DIAZ-VAZ, J.E. 2003. Anatomía de Maderas. Marisa Cœneo Ediciones, Chile 151 p.

DUCHESNE, I.; ZHANG, SY. 2004. Variation in tree growth, wood density, and pulp fiber properties of 35 white spruce (Picea glauca (Moench) Voss) families grown in Quebec. Wood and Fiber Science 36(4):467-475.

Du TORR,A. 1963. A study of the influence of compression wood on the warping of Pinus radiata D. Don timber. S. Afr.For.J. 44:11-15.

EVANS, R. 1994 Rapid measurement of the transverse dimensions of tracheids in radial wood sections from Pinus radiata. Holzforschung (48):168-172.

DONALDSON, L.; GRACE, J.; DOWNES, G. 2004. Within-tree variation in anatomical properties of compression wood in radiata pine. IAWA Journal 25(3):253-271.

HARRIS, M. 1977. Shrinkage and density of radiata pine compression wood in relations to its anatomy and mode of formation. New Zealand Journal of Forestry Science 7(1):91-106

JONES, P.; SCHIMLECK, L.; PETER, G.; DANIELS, R.; CLARK III,A. 2005. Non-destructive estimation of Pinus taeda L. tracheid morphological characteristics for samples from a wide range of sites in Georgia. Wood Sci. Technol. 39: 529-545.

LINDSTRÖM, H. 1997. Fiber length, tracheid diameter, and laterwood percentage in Norway spruce : Development from pith outwards. Wood and Fiber Science 29(1):21-34.

LINDSTRÖM, H.; HARRIS, P.; SORENSSON, C. 2004. Stiffness and wood variation of 3-year old Pinus radiata clones. Wood Sci. Technol. 38:579-597.

LOMAGNO, J.; ROZAS, C. 2001 Determinación de la madera de compresion en Pinus radiata D. DON. Maderas. Ciencia y tecnología 3(1-2):63-67.

LOW, A. 1964. Compression wood in conifers a review of literature. Forestry Abstracts 25(3):35-43

MARTINEZ, G.; PACHECO, G.; SANDOVAL, F. 1989. Pinus radiata D.Don: caracterización, bondades y potencialidades. V Congreso Latinoamericano de la Celulosa y el Papel, Santiago de Chile. 19$28 \mathrm{pp}$.

MAYR, S.; COCHARD, H. 2003. A new method for vulnerability analysis of small xylem areas reveals that compression wood of norway spruce has lower hydraulic safety than opposite wood. Plant, Cell and Environment 26: 1365-1371. 
MELO, R.; PAZ, J.; CARRASCO, V.; BELLO, N. 1988. Interrelación entre las propiedades de una celulosa kraft y la materia prima usada para su fabricación. Celulosa y el Papel (4) 3: 15-20

MOÉLL, M.; FUJITA, M. 2004. Fourier transform methods in image analysis of compression wood at the cellular level. IAWA Journal 25(3): 311-324.

MOLTENBERG, D.; MØIBØ, O. 2006. Development and variation of wood density, kraft pulp yield and fiber dimensions in young norway spruce (Picea abies). Wood Sci. Technol (40): 173-189.

MORALES, E. 2005. Diseño experimental a través del análisis de varianza y modelo de regresión lineal. Ed. Carouna. Santiago Chile 248 p.

MUNERI, A.; RAYMOND, C. 2001. Nondestructive sampling of Eucalyptus globulus and E. nitens for wood properties; II Fiber length and coarseness. Wood Sci. Technol. 35:41-56.

NYAKUENGAMA, J.; DOWNES, G.; NG, J. 2003. Changes caused by mid-rotation fertilizer application to the fibre anatomy of Pinus radiata. IAWA Journal 24(4):397-409.

NICHOLLS, J. 1982. Wind action, leaning trees and compression wood in Pinus radiata D.Don. Aust. For. Res.12:75-92.

ÖHMAN, M. 1999 Correspondences between manually estimated compression wood in norway spruce and the warp of the sawn timber. Holz als Roh-und Werkstoff 57(5):391-396.

RIVAS, C. 1982 Determinación de madera juvenil en Pinus radiata D.Don. Tesis Ingeniero Forestal, Valdivia Chile. Fac. Cs. Forestales, Universidad Austral de Chile. 76 p.

ROFFAEL, E.; ESSIAMAH, S.; DIAZ-VAZ, J.E.; SCHNEIDER, T.; DIX, B. 2005. Untersuchungen über den Einflu§ von Reaktionsholz (Druckholz) und Normalholz der Fichte auf die Eigenschaften von mitteldichten Faserplatten (MDF). Forstarchiv (76): 206-214.

SCHIMLECK, L.; EVANS, R. 2004 Estimation of Pinus radiata D.Don tracheid morphological characteristics by near infrared spectroscopy. Holzforschung 58:66-73.

SCHMITT, U.; SINGH, A.P.; THIEME, H.; FRIEDRICH, P.; HOFFMANN. P. 2005. Electron microscopic characterization of cell wall degradation of the 400,000-year-old wooden Schoningen spears. Holz als Roh-und Werkstoff 63(2): 118-122.

SINGH,A. 1997. The ultrastructure of the attack of Pinus radiata mild compression wood by erosion and tunnelling bacteria. Can. J. Bot. 75: 1095-1102.

SINGH, A.; DONALDSON, L.A. 1999. Ultrastructure of tracheid cell walls in radiata pine (Pinus radiata) mild compression wood. Can. J. Bot. 77:32-40.

SHELBOURNE, C.; RITCHIE, K. 1968. Relationships between degree of compression wood development an specific gravity and tracheid characteristics in loblolly pine (Pinus taeda L.). Holzforschung 22(6): 185-190.

SPICER, R.; GARTNER, B. 1998. Hydraulic properties of douglas-fir (Pseudotsuga menziesii) branches and branch halves with reference to compression wood. Tree Physiology 18:777-784. 
TIMELL, T. 1982. Recent progress in the chemistry and topochemistry of compression wood. Wood Sci. Technol. 16:83-122.

TIMELL, T. 1986. Compression Wood in Gymnosperms Vol. 1-3, Springer-Verlag, Berlin 2150 pp.

TRENDELENBURG, R.; MAYER-WEGELIN, H. 1955. Das Holz als Rohstoff. Carl Hanser Verlag, München 140:143 pp

UPRICHARD, J. 1980. Effects of wood age on the papermaking properties of radiate pine kraft pulps. New Zealand Journal of Forestry Science 10(3): 558-576

UPRICHARD, J. 2002 Pulp and paper from radiata pine. Appita and Forest Research, Australia, 478p.

VARGAS, G.; DIAZ-VAZ, J.E. 1992. Densidad básica y ancho de anillo afectados por madera de compresión en pino radiata crecido en Valdivia-Chile. Pinus radiata investigación en Chile, Facultad de Ciencias Forestales Universidad Austral de Chile, Valdivia, 324-329 pp.

WATSON, P.; GARNER, C.; RBERTSON, R.; REATH, S.; GEE, W.; HUNT, K. 2003. The effects of initial tree spacing on the fibre properties of plantation-grown coastal western hemlock. Can.J.For.Res. $33: 2460-2468$.

YOSHIZAWA, N.; KIYOMIYA, M.; IDEI, T. 1987. Variations in tracheid length and morphological changes in tracheid tips associated with the development of compression wood. Wood Sci. Technol. 21(1):110.

ZOBEL, B. 1981. Wood quality from fast-grown plantations. TAPPI 64(1):71-74. 
